\title{
Reduced thermal dependence of the sensitivity of a planar Hall sensor
}

DOI:

$10.1063 / 1.5110671$

\section{Document Version}

Final published version

Link to publication record in Manchester Research Explorer

\section{Citation for published version (APA):}

Mahfoud, M., Tran, Q-H., Wane, S., Ngo, D-T., Belarbi, E. H., Boukra, A., Kim, M., Elzwawy, A., Kim, C., Reiss, G., Dieny, B., Bousseksou, A., \& Terki, F. (2019). Reduced thermal dependence of the sensitivity of a planar Hall sensor. Applied Physics Letters, 115(7). https://doi.org/10.1063/1.5110671

\section{Published in:}

Applied Physics Letters

\section{Citing this paper}

Please note that where the full-text provided on Manchester Research Explorer is the Author Accepted Manuscript or Proof version this may differ from the final Published version. If citing, it is advised that you check and use the publisher's definitive version.

\section{General rights}

Copyright and moral rights for the publications made accessible in the Research Explorer are retained by the authors and/or other copyright owners and it is a condition of accessing publications that users recognise and abide by the legal requirements associated with these rights.

\section{Takedown policy}

If you believe that this document breaches copyright please refer to the University of Manchester's Takedown Procedures [http://man.ac.uk/04Y6Bo] or contact uml.scholarlycommunications@manchester.ac.uk providing relevant details, so we can investigate your claim.

\section{OPEN ACCESS}




\section{Reduced thermal dependence of the sensitivity of a planar Hall sensor}

Cite as: Appl. Phys. Lett. 115, 072402 (2019); https://doi.org/10.1063/1.5110671

Submitted: 21 May 2019. Accepted: 31 July 2019. Published Online: 12 August 2019

Mohamed Mahfoud (D), Quang-Hung Tran (D), Sidina Wane, Duc-The Ngo (D), El Habib Belarbi (D), AbdelAziz Boukra, Mijin Kim, Amir Elzwawy (D), CheolGi Kim (D), Guenter Reiss (D), Bernard Dieny (D), Azzedine Bousseksou, and Ferial Terki (D)
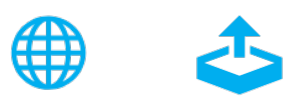

\section{ARTICLES YOU MAY BE INTERESTED IN}

Molecular beam epitaxy growth of antiferromagnetic Kagome metal FeSn

Applied Physics Letters 115, 072403 (2019); https://doi.org/10.1063/1.5111792

High frequency voltage-induced ferromagnetic resonance in magnetic tunnel junctions Applied Physics Letters 115, 072401 (2019); https://doi.org/10.1063/1.5113681

Tuning the shape of magneto-optical Kerr spectrum by changing the strip width in onedimensional Ag-Co-Ag magnetoplasmonic nanogratings

Applied Physics Letters 115, 072405 (2019); https://doi.org/10.1063/1.5096912

\section{Applied Physics Letters}

Mid-IR and $\mathrm{THz}$ frequency combs special collection

Read Now! 


\title{
Reduced thermal dependence of the sensitivity of a planar Hall sensor
}

\author{
Cite as: Appl. Phys. Lett. 115, 072402 (2019); doi: 10.1063/1.5110671 \\ Submitted: 21 May 2019 . Accepted: 31 July 2019 . \\ Published Online: 12 August 2019
}

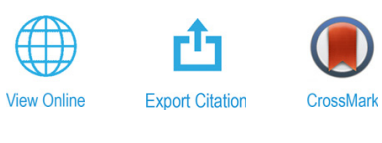

Mohamed Mahfoud, ${ }^{1,2}$ (iD Quang-Hung Tran,, ${ }^{3, a)}$ (D Sidina Wane, ${ }^{3}$ Duc-The Ngo, ${ }^{4}$ (iD El Habib Belarbi, ${ }^{2}$

AbdelAziz Boukra, ${ }^{5}$ Mijin Kim, ${ }^{6}$ Amir Elzwawy, ${ }^{6}$ (iD CheolGi Kim, ${ }^{6}$ (D) Guenter Reiss, ${ }^{7}$ (iD Bernard Dieny, Azzedine Bousseksou, ${ }^{9}$ and Ferial Terki ${ }^{1, a)}$ (iD

\author{
AFFILIATIONS \\ 'Institut Charles Gerhardt Montpellier, UMR 5253 UM-CNRS-ENSM, Ingénierie Moléculaire et Nano-Objets, \\ Université de Montpellier, Place E. Bataillon, 34095 Montpellier, France \\ ${ }^{2}$ Synthesis and Catalysis Laboratory LSCT, Tiaret University, 14000 Tiaret, Algeria \\ ${ }^{3}$ Sensors R\&D\&C, eV-Technologies, 14000 Caen, France \\ ${ }^{4}$ Electron Microscopy Centre, Department of Materials, University of Manchester, Oxford Road, Manchester M13 9PL, United Kingdom \\ ${ }^{5}$ Laboratoire de Structure, Élaboration et Application des Matériaux Moléculaires, SEA2M, Université Abdelhamid Ibn Badis, \\ 27000 Mostaganem, Algeria \\ ${ }^{6}$ Department of Emerging Materials Sciences, DGIST-Daegu Gyeongbuk Institute of Science and Technology, Daegu 42988, \\ South Korea \\ ${ }^{7}$ Department of Physics, Universität Bielefeld, P.O. Box 100 131, 33501 Bielefeld, Germany \\ ${ }^{8}$ Grenoble Alpes University, CEA, CNRS, Grenoble INP, IRIG, SPINTEC, 38054 Grenoble, France \\ ${ }^{9}$ Laboratoire de Chimie de Coordination UPR 8241, CNRS, 31007 Toulouse Cedex 4, France
}

${ }^{a)}$ Authors to whom correspondence should be addressed: quang-hung.tran@ev-technologies.com and ferial.terki@umontpellier.fr

\begin{abstract}
The ability to stabilize the sensitivity of a magnetoresistance sensor in unstable thermal environments is a key parameter in many high precision measurements. Here, we propose a method to stabilize the sensitivity of a highly sensitive and low noise magnetic sensor based on a planar Hall Effect crossed junction. The stability is achieved by controlling the interplay between Zeeman energy, exchange bias energy, and anisotropy energy as a function of the temperature of the sensor stack comprising a trilayer structure $\mathrm{NiFe} / \mathrm{Cu} / \mathrm{IrMn}(10 / 0.12 / 10 \mathrm{~nm})$. The high thermal stability of the sensor sensitivity of $4.5 \pm 0.15 \times 10^{-3} \mathrm{~V} / \mathrm{A} / \mathrm{T} / \mathrm{K}$ is achieved when the external magnetic field is set around $\pm 2 \pm 0.04 \mathrm{mT}$ and the applied current is fixed at $20 \mathrm{~mA}$ in the temperature range of $110 \mathrm{~K}-360 \mathrm{~K}$. This method improves the magnetic sensor detection by about an order of magnitude, enabling its deployment in various research fields, particularly to study magnetic properties of small quantities of magnetic materials toward the detection of single magnetic objects, which was impossible before.
\end{abstract}

Published under license by AIP Publishing. https://doi.org/10.1063/1.5110671

The thermal drift of the magnetoresistive sensor sensitivity strongly affects the accuracy of magnetic measurements. However, many emerging applications require the use of a sensor in a wide range of temperature environments from extremely cold to furnace temperatures, such as aerospace, ${ }^{1}$ automotive, ${ }^{2}$ and biochips, ${ }^{3,4}$ and especially, in our recent patented ultrasensitive subfemto $\mathrm{Am}^{2}$ micromagnetometer. ${ }^{5,6}$ The micromagnetometer is based on the Planar Hall Effect (PHE). Under an external magnetic field, the magnetization of the sensor stack is oriented toward the field direction, inducing the change in the longitudinal and the transversal resistivities $\left(\rho_{\mathrm{xx}}\right.$ and $\left.\rho_{\mathrm{xy}}\right)$. The variation of the transversal resistivity $\left(\rho_{\mathrm{xy}}\right)$ is called the PHE, whereas the variation of the longitudinal resistivity $\left(\rho_{\mathrm{xx}}\right)$ is called the anisotropic magnetoresistive (AMR) effect. ${ }^{7}$

The magnetometer operating in a temperature range of $200 \mathrm{~K}$ to $400 \mathrm{~K}$ was employed for characterizing the spin-switching from the low spin state (diamagnetic) to the high spin state (paramagnetic) of the spin-crossover materials with a small magnetic moment of $10^{-17} \mathrm{Am}^{2}$. The related results were highlighted recently in Angew. Chem. Int. Ed. ${ }^{8}$ and Adv. Mater. ${ }^{9}$ However, the sensitivity variation with the temperature of this sensor defined as $\mathrm{d} S / \mathrm{d} T$ (with $S$ being the 
sensitivity and $T$ the temperature) is about $0.05 \mathrm{~V} / \mathrm{A} / \mathrm{T} / \mathrm{K}$ when the applied current was fixed at $20 \mathrm{~mA}$. Reduction of this high temperature dependence will result in a more accurate detection, enabling the sensor to measure physical properties of magnetic materials at the nanoscale.

In this letter, we report a method to stabilize the sensitivity of a PHE sensor in an unstable thermal environment. The ultrahigh sensitivity of this sensor is optimized by controlling the exchange bias coupling via an atomic $\mathrm{Cu}$ sublayer in a tailored exchange bias structure $\mathrm{NiFe} / \mathrm{Cu} / \mathrm{IrMn} .^{10}$ A low white noise of $1 \mathrm{nT} / \sqrt{\mathrm{Hz}}$ could be reached for this sensor. We demonstrate that by adjusting the temperature dependences of the exchange bias and anisotropy while considering those of the magnetization and AMR amplitude, the sensitivity of a PHE sensor can be made quite stable with a maximum variation of $4.5 \pm 0.15 \times 10^{-3} \mathrm{~V} / \mathrm{A} / \mathrm{T} / \mathrm{K}$ within the studied temperatures. The temperature coefficient of the sensitivity obtained in this work $(0.02 \% / \mathrm{K})$ is lower than that achieved in a reported PHE sensor (a deduced value of $0.25 \% / \mathrm{K}){ }^{11}$ a giant magnetoresistive (GMR) sensor $(-0.13 \% / \mathrm{K}){ }^{12}$ and an AMR sensor $(0.1 \% / \mathrm{K})^{13}$ and comparable to the temperature dependence of an optimized tunnel magnetoresistive (TMR) sensor $(0.01 \% / K){ }^{14}$ Note that in previous studies, the temperature range is smaller than the temperature range in this study. In order to explain the thermal stability of the sensitivity, we investigate the temperature dependence of various parameters of the sensor stack such as the exchange bias field $\left(H_{\mathrm{ex}}\right)$, coercive field $\left(H_{\mathrm{c}}\right)$, anisotropic resistivity $(\Delta \rho)$, and easy axis angle. The achievement of this sensitive stability enables us to use the sensor for many precise measurements in an unstable thermal environment without the need of a correction tool. Specifically, in our subfemto $\left(10^{-17}\right) \mathrm{Am}^{2}$ micromagnetometer, ${ }^{5,9}$ it could improve the detection limit by one order of magnitude, down to atto $\left(10^{-18}\right) \mathrm{Am}^{2}$.

Figure 1(a) shows a PHE sensor junction of $50 \mu \mathrm{m} \times 50 \mu \mathrm{m}$ and the trilayer stack $\mathrm{NiFe}(10 \mathrm{~nm}) / \mathrm{Cu}(0.12 \mathrm{~nm}) / \mathrm{IrMn}(10 \mathrm{~nm})$ with $5 \mathrm{~nm}$ Ta capping and seed layers deposited on the $\mathrm{Si} / \mathrm{SiO}_{2}(625 / 0.5 \mu \mathrm{m})$ substrate using a DC sputtering system [Daegu vacuum, South Korea] [fabrication conditions are provided in S1]. Figure 1(b) shows the room temperature $M-H$ hysteresis loop of the stack. The loop shifted along the field axis due to the exchange interaction between the NiFe ferromagnetic (FM) and the IrMn antiferromagnetic (AFM) layers. The thin film cross section was imaged using a high-resolution transmission electron microscopy (HRTEM) system. In the HRTEM image [Fig. 1(c)], both the Ta layers appear as amorphous, NiFe, and IrMn layers have a polycrystalline structure denoted by lattice fringes. Lattice fringes characteristic of IrMn (e.g., (101)-0.22 nm) and NiFe (e.g., (111) $-0.19 \mathrm{~nm}$ ) can be clearly seen from the HRTEM image [the details are described in the Fig. 1(c), (1) and (2) insets]. In addition, the thicknesses of $\mathrm{NiFe}$, IrMn, and Ta are determined by averaging 10 measurements at different areas along the cross-sectional view. Their respective values are $10.0 \pm 0.5 \mathrm{~nm}, 10.6 \pm 0.6 \mathrm{~nm}$, and $5 \pm 0.25 \mathrm{~nm}$. Despite these precise quantifications, the nominal $\mathrm{Cu}$ atomic layer of $0.12 \mathrm{~nm}$ sandwiched between $\mathrm{NiFe}$ and IrMn layers could not be visible due to the resolution limit of HRTEM imaging and the close similarity between the atomic weight of $\mathrm{Cu}$ and $\mathrm{NiFe}$ and lattice parameters.

To determine the temperature dependence of the sensitivity, the sensor was placed in a temperature controller (Linkam LTS 420, UK) to isolate it from external factors. To investigate the electrical
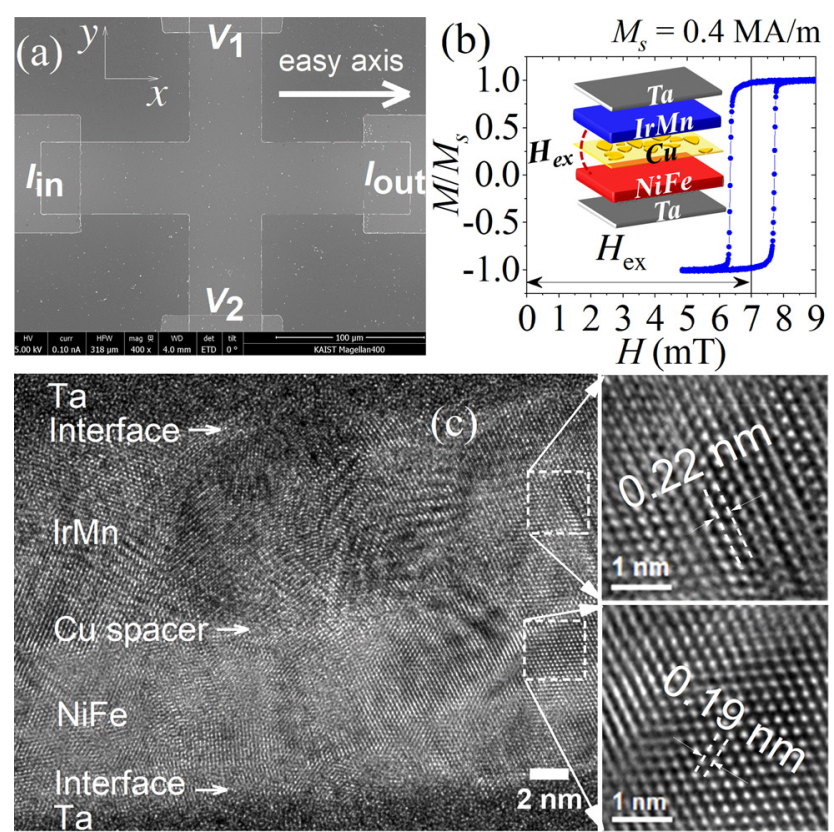
$H(\mathrm{mT})$

FIG. 1. (a) A top view SEM image of a crossed junction of the sensor (the bar width is $50 \mu \mathrm{m}$; easy axis, electrodes for applied current and measured voltage of the junction are indicated). (b) Room temperature $\mathrm{M}-\mathrm{H}$ hysteresis loop of the thin film; the saturation magnetization $M_{s}=0.4 \mathrm{MA} / \mathrm{m}$. (c) Cross-sectional HRTEM image of the thin film stack $\mathrm{Ta}(5 \mathrm{~nm}) / \mathrm{NiFe}(10 \mathrm{~nm}) / \mathrm{Cu}(0.12 \mathrm{~nm}) / \mathrm{rMn}(10 \mathrm{~nm}) / \mathrm{Ta}(5 \mathrm{~nm})$. The TEM specimen was fabricated using a focused ion beam (FIB, Hitachi NB 5000). The insets (1) and (2) show zoomed areas from NiFe and IrMn layers where the electron beam went through $\langle 101\rangle$ and $\langle 111\rangle$ zone axes of IrMn and NiFe nanocrystals, respectively.

responses of the sensor, a current of $20 \mathrm{~mA}$ was applied in the direction of the sensor's easy axis. An AC external magnetic field was applied in two directions, perpendicular and parallel to the easy axis. The voltage profiles for these two cases were measured by using a lock-in amplifier [SR830, Stanford Research Systems, US] at a frequency of $113.7 \mathrm{~Hz}$.

Figures 2(a) and 2(b) show the measured and calculated PHE voltage profiles when the external magnetic field was applied perpendicular and parallel to the sensor's easy axis, respectively, at $T=270 \mathrm{~K}$.

Under an external magnetic field, the electrical response of the sensor stack is given by the following equation: ${ }^{10}$

$$
V_{\mathrm{PHE}}=\frac{I\left(\rho_{/ /}-\rho_{\perp}\right)}{t} \cos (\theta) \sin (\theta),
$$

where $I$ is the current applied to the sensor junction, $t$ is the thickness of the ferromagnetic layer, $\rho_{/ /}$and $\rho_{\perp}$ are the longitudinal and transverse components of the resistivity, and $\theta$ is the angle between the magnetization and the current direction applied parallel to the easy axis of the sensor stack.

Angle $\theta$ is calculated by the energy minimum condition of the stack that involves three terms: Anisotropy energy, Zeeman energy, and exchange bias energy between the FM and AFM layers, ${ }^{10}$

$$
E=K_{u} t \sin ^{2}(\theta)-M_{s} t H \cos (\alpha-\theta)-M_{s} t H_{e x} \cos (\theta),
$$



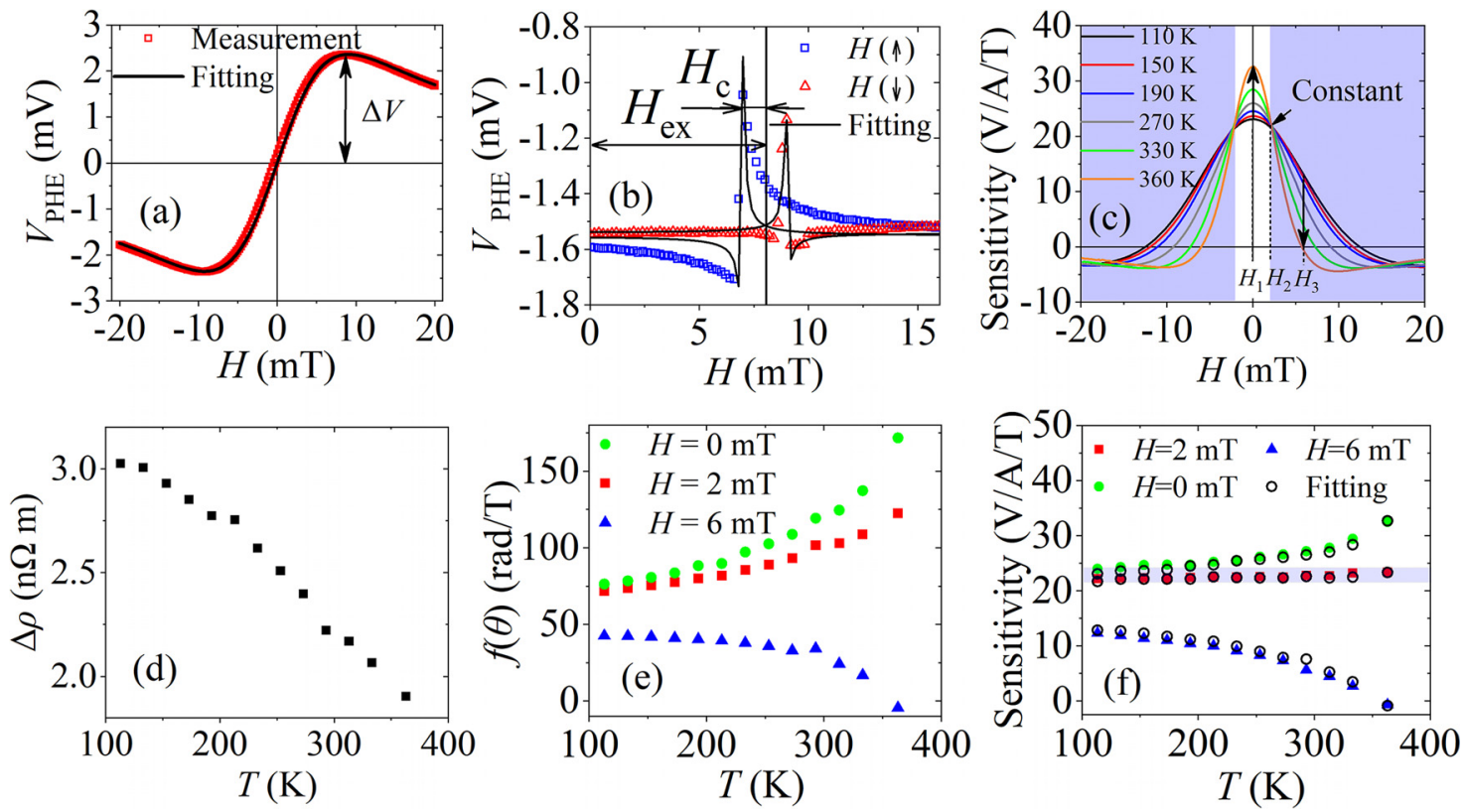

FIG. 2. (a) and (b) Magnetotransport measurements via planar Hall voltage profiles for the magnetic field applied in plane of the sensor junction along the hard axis (y-axis) and easy axis (x-axis), respectively at $T=270 \mathrm{~K}$. (c) Variation of the field sensitivity as a function of the external magnetic field at various temperatures (from $110 \mathrm{~K}$ to $360 \mathrm{~K}$ ) with $\alpha=90^{\circ}$. (d) Anisotropic resistivity of the sensor as a function of temperature (from $110 \mathrm{~K}$ to $360 \mathrm{~K}$ ) calculated from the fitting curves. (e) Calculated $f(\theta)$ as a function of temperature from $110 \mathrm{~K}$ to $360 \mathrm{~K}$. (f) Variation of the field sensitivity deduced from the measured $V_{\mathrm{PHE}}(H)$ and calculated ones from Eq. (3) as a function of temperature for three different fields $H=0 \mathrm{mT}, 2 \mathrm{mT}$, and $6 \mathrm{mT}$.

where $K_{\mathrm{u}}$ is the effective anisotropy constant, $t$ is the thickness of the FM layer, $M_{\mathrm{s}}$ is the saturation magnetization of the FM layer, $H$ is the external magnetic field, $\alpha$ is the angle between the easy axis of the sensor and the external magnetic field axis, and $H_{\mathrm{ex}}$ is induced by the interfacial interaction between the FM and AFM layers.

The energy minimization is performed using the NewtonRaphson numerical method based on an iterative process. ${ }^{15}$ To obtain parameters of the sensor such as $H_{\mathrm{ex}}, H_{\mathrm{c}}, \Delta \rho$, and the easy magnetization angle, the experimental $V_{\mathrm{PHE}}(H)$ profiles were fitted by Eq. (1) using the nonlinear least squares method. ${ }^{15}$

When the applied magnetic field is perpendicular to the easy magnetization axis, the PHE voltage increases monotonically with the magnetic field until the field reaches its extremum value for $\theta=\pi / 4$. A further increase in the field beyond the extremum value induces a decrease in the PHE voltage since the PHE voltage varies as $\sin 2 \theta$ [Fig. 2(a)].

When the applied magnetic field is parallel to the easy magnetization axis, the exchange interaction between the FM and AFM layers pins the magnetic moments of the FM layer in its direction. $V_{\mathrm{PHE}}$ exhibits two through peaks, one along the ascending branch of the hysteresis loop and another one along the descending branch of the loop. These peaks are centered at $H_{\mathrm{c}}$ since they occur during the magnetization reversal. The loop is shifted along the field axis, which is the characteristic of the exchange bias phenomenon. At an external field value around $H_{\mathrm{ex}}$, the magnetization of the NiFe layer is broken into a set of small irregular domains, which expand quickly to complete the magnetization reversal when the field further increases. ${ }^{16}$ As a result, the $V_{\mathrm{PHE}}$ signal returns to its low value when the magnetization reversal is completed [see Fig. 2(b)].
The fitted sensitivity profiles $S(H)$ were obtained by fitting the experimental $S(H)$ at various temperatures using Eq. (3) [See supplementary material S2 for details]. Here, for all temperatures, the field sensitivity $S(H)$ profiles have a maximum value at zero field $\left(H_{1}\right)$, decrease down to zero when the magnetic fields satisfied $\theta=\pi / 4$, and become negative for $\theta>\pi / 4$.

The field sensitivity varies differently as a function of temperature in three ranges of magnetic fields. (1) In the field range of $-2 \mathrm{mT}$ to $+2 \mathrm{mT}$, the field sensitivity increases with temperature.(2) Outside of this field interval (i.e., $H$ in the ranges of $-20 \mathrm{mT}$ to $-2 \mathrm{mT}$ and $+2 \mathrm{mT}$ to $+20 \mathrm{mT}$ ), the field sensitivity decreases with temperature. (3) At $H= \pm 2 \mathrm{mT}$, the field sensitivity is almost independent of temperature over the investigated temperature range.

To clearly understand these three different behaviors of the field sensitivity vs temperature in correlation with physical parameters of the sensor, we rewrite the field sensitivity term $\mathrm{d} V_{\mathrm{PHE}} / \mathrm{d} H$ as shown in the following equation:

$$
S(H)=\frac{I_{\left(\rho_{/ /}-\rho_{\perp}\right)}}{t} \cos (2 \theta) \frac{d \theta}{d H} .
$$

In Eq. (3), $S(H)$ can be written as the product of $I / t \times \Delta \rho\left(\Delta \rho=\rho_{\text {// }}\right.$ $\left.-\rho_{\perp}\right)$ and $f(\theta)=\cos (2 \theta) \mathrm{d} \theta / \mathrm{d} H$ functions. Thus, we analyze these two functions vs temperature to understand the three different behaviors of the field sensitivity mentioned above. This analysis is performed at three different fields $H=0 \mathrm{mT}, 2 \mathrm{mT}$, and $6 \mathrm{mT}$ representing for the three regimes as discussed previously [see supplementary material S3 for details]. Note that except the role of the resistivity discussed separately, all other intrinsic and 

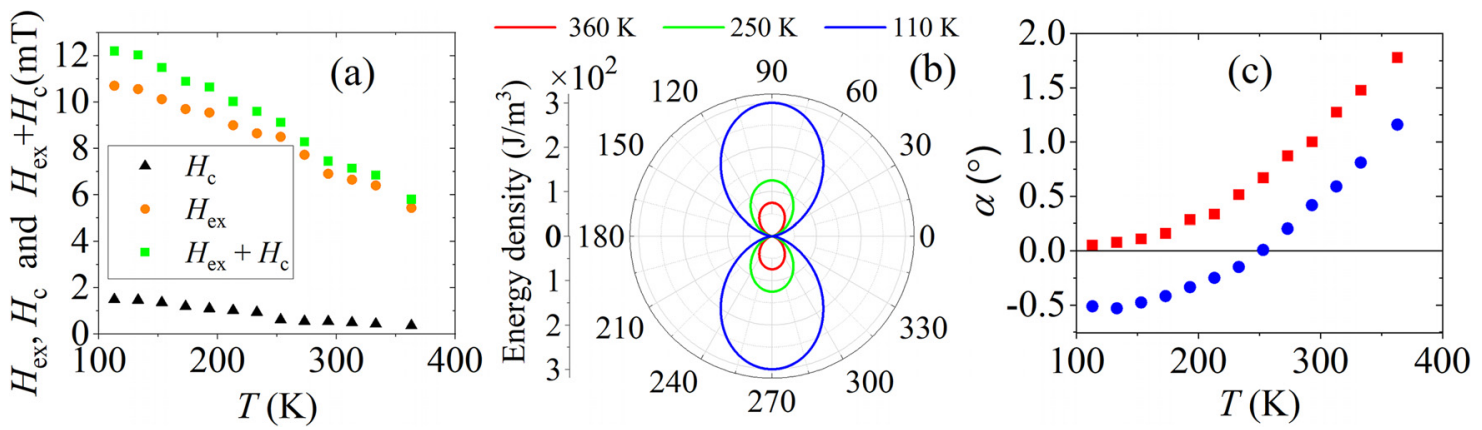

FIG. 3. (a) The calculated exchange bias, coercive fields, and their sum of the investigated magnetic stack as a function of temperature in the range of $110 \mathrm{~K}$ to $360 \mathrm{~K}$. (b) The variation of the anisotropy energy of the sensor stack as a function of the angle $\theta$ at three temperatures of $110 \mathrm{~K}$, $250 \mathrm{~K}$, and $360 \mathrm{~K}$ (The anisotropy energy was calculated for the magnetic field applied in plane of the sensor junction along the hard magnetization axis). (c) The change in the easy axis angle with temperature in the range of $110 \mathrm{~K}$ to $360 \mathrm{~K}$ for two measurement conditions corresponding to the external field aligned with the easy axis direction at $110 \mathrm{~K}$ and at $250 \mathrm{~K}$.

extrinsic parameters that affected the sensitivity are included and discussed in this $f(\theta)$ function.

Figure 2(d) shows the anisotropic resistivity $\Delta \rho$ as a function of the temperature from $110 \mathrm{~K}$ to $360 \mathrm{~K}$. In agreement with previous results on $\mathrm{NiFe}$ anisotropic magnetic resistivity, ${ }^{17}$ we observed a decrease in the amplitude of $V_{\text {PHE }}$ profiles vs temperature within the investigated temperature range [See S4 for clarification].

Figure 2(e) represents the temperature dependence of the value taken by the function $f(\theta)=\cos (2 \theta) \mathrm{d} \theta / \mathrm{d} H$ for the three different external magnetic fields $H=0 \mathrm{mT}, 2 \mathrm{mT}$, and $6 \mathrm{mT}$ mentioned above. At $H=0 \mathrm{mT}$, the function $f(\theta)$ rapidly increases from $76.3 \mathrm{rad} / \mathrm{T}$ to $171.7 \mathrm{rad} / \mathrm{T}$ within the temperature range of $110 \mathrm{~K}-360 \mathrm{~K}$. At $H=2 \mathrm{mT}$, the increment of $f(\theta)$ becomes slower than its value at $H=0 \mathrm{mT}$. In contrast, the function $f(\theta)$ decreases with the temperature when the external magnetic field $H$ is at $6 \mathrm{mT}$. These various behaviors of $f(\theta)$ at different fields result from the variations of the coercive field, exchange bias field, and easy axis direction with temperature [see supplementary material S5 for the full description of the $f(\theta)$ function].

Figure 2(f) shows the variation of the sensitivity as a function of temperature. Here, we again observe three different behaviors of the field sensitivity for the three selected field values. These include a monotonic increase with temperature at $0 \mathrm{mT}$, a gradual decrease with temperature at $6 \mathrm{mT}$, and stable behavior within the studied temperature range at $2 \mathrm{mT}$. These experimental variations deduced from the measurement of $V_{\mathrm{PHE}}(H)$ profiles are in good agreement with our calculations using Eq. (3).

In this later case, the interplay between electric and magnetic properties of the sensor represented by $\Delta \rho$ and $f(\theta)$ induces the thermal stability of the sensor sensitivity, respectively. Such a stable thermal behavior of the field sensitivity is crucial for both fundamental and applied interest of a magnetic sensor operating in variable temperature environments.

The thermal stability of the field sensitivity is governed by the interplay between electrical and magnetic parameters of the thin film as explained above. Concerning the magnetic one, they are the magnetization, the anisotropy field, and the exchange bias field. In the following, we discuss the behavior of these parameters regarding the fields $H_{\mathrm{c}}$ and $H_{\mathrm{ex}}$ and the easy axis angle $(\alpha)$ in the investigated temperature range. The dependence of these three variables on the temperature is shown in Fig. 3.
Figure 3(a) shows the variation of $H_{\mathrm{ex}}, H_{\mathrm{c}}$, and their sum extracted from the fitting of the measured $V_{\mathrm{PHE}}(H)$ curves as a function of the temperature [See supplementary material S6 for $V_{\mathrm{PHE}}(H)$ curves and the method of deduction]. It is clear that both $H_{\mathrm{ex}}$ and $H_{\mathrm{c}}$ increase as the temperature decreases. The decrease in these two physical parameters is related to the interactions between the FM and AFM layers, which pins the magnetic moments in the FM layer and also affects the domain wall mobility in the FM layer. ${ }^{16}$ At low temperatures, the spin lattice of the IrMn layer is more strongly frozen, ${ }^{18}$ which induces a stronger interaction between the moments in the AFM layer and those in the FM layer than that at high temperature. As a result, the exchange bias and the coercive field increase when decreasing temperature.

Figure 3(b) shows the variation of the anisotropic energy density as a function of the angle $\theta$ at selected temperatures $(T=360 \mathrm{~K}, 250 \mathrm{~K}$, and $110 \mathrm{~K})$. Obviously, in all cases, the anisotropy energy shows two energy minima corresponding to the two easy magnetization directions of the sensor. In fact, the anisotropy energy decreases with the increase in the temperature due to the weak interaction between the FM and the AFM layers. The switch of the magnetization between the two energy minima is easier at high temperature than the switch at low temperature. ${ }^{19}$

Intrinsic rotation of the easy axis from its referenced direction $(\alpha)$ vs temperature is another factor contributing to the temperature variation of $f(\theta)$ as discussed in Fig. 2(e). We note that a slight misalignment between the easy axis direction and the external magnetic field direction could affect the reversal mechanism of the FM layer magnetization. ${ }^{16}$ Therefore, we study the effect of the temperature on the easy axis direction as illustrated in Fig. 3(c). Two configuration conditions are considered, the first one corresponds to the alignment of the external field with the easy axis $\left(\alpha=0^{\circ}\right)$ at low temperature $(T=110 \mathrm{~K})$, while the second one corresponds to the alignment of the external magnetic field with the easy axis at high temperature $(T=250 \mathrm{~K})$. The values of angle $\alpha$ are extracted from the fitting of the measured $V_{\mathrm{PHE}}(H)$ curves at various temperatures by using Eq. (1). The fitting is done using the nonlinear least squares method, and the angle $\theta(H)$ is obtained by the minimization of the energy described in Eq. (2).

In these two experimental conditions, the easy magnetization direction aligned with the external magnetic field at $T=110 \mathrm{~K}$ and $T=250 \mathrm{~K}$, the deviation of the easy axis from its referenced direction 
with temperature, $\alpha(T)$, has a similar nonlinear increasing trend. Quantitatively, the angle $\alpha$ varies by about $1.7^{\circ}$ as the temperature increases within the temperature range of $110 \mathrm{~K}-360 \mathrm{~K}$ (from $0^{\circ}$ to $1.78^{\circ}$ in the first condition and from $-0.51^{\circ}$ to $+1.16^{\circ}$ in the second condition). These results indicate that the orientation of the easy axis is slightly influenced by the temperature possibly due to the slight magnetostrictive effect induced by the presence of the IrMn layer.

In summary, we have given a detailed description of a PHE sensor's behavior in the range of temperatures from $110 \mathrm{~K}$ to $360 \mathrm{~K}$. The physical properties of a PHE sensor such as the exchange bias, coercive field, and the anisotropic resistivity decrease with increasing temperature. However, the study of the effect of the temperature on the easy magnetization axis shows the existence of a small rotation $\left(1.7^{\circ}\right)$ from its referenced axis, which can slightly influence the sensor's behavior regarding the reversal mechanism of magnetization. These investigations enable us to understand the mechanisms governing the high thermal stability of the sensor's sensitivity. By sweeping a wide range of the applied magnetic field from $-20 \mathrm{mT}$ to $+20 \mathrm{mT}$, we found that $2 \mathrm{mT}$ corresponds to the optimal stability of the sensitivity with an extremely low variation of $4.5 \pm 0.15 \times 10^{-3} \mathrm{~V} / \mathrm{A} / \mathrm{T} / \mathrm{K}$. This is the lowest temperature drift amplitude of the sensitivity which has never been achieved hitherto within a quite large temperature range [110 K-360 K] compared to other thin film based magnetoresistive sensors. Finally, the control of the temperature stability of the sensitivity could be a key parameter in emerging applications such as nanosatellite, next generation of biological chip, and automotive applications. Specifically, it is important in the development of our magnetometer with a detection resolution lower than $10^{-18} \mathrm{Am}^{2}$. This improvement will be beneficial for the characterization of low volume and low dimension magnetic materials with small susceptibilities such as bistable materials and single molecular magnets.

See the supplementary material for the information of the fabrication of a PHE sensor [S1], information about the variation of $S(H)$ at different temperatures [S2], the calculation of the thermal stability of the sensor sensitivity [S3], $V_{\mathrm{PHE}}(H)$ profiles when the magnetic field is applied along the hard axis at different temperatures to highlight the decrement of its amplitude with temperature [S4], The expression of $f(\theta)$ is clarified in [S5], and the deduced exchange bias fields and coercive fields obtained from the $V_{\mathrm{PHE}}(H)$ profiles when the magnetic field is applied along the easy axis for different temperatures [S6].
This work was supported by the Algerian-French program PROFAS B + of the Ministry of Higher Education and Scientific Research, Directorate of Cooperation and inter-university exchange, the French Institute of Algeria (Algeria), the Scientific and Innovative Research Investment Program, Prematuration HEMOSENS-2017-006697 of the Occitanie Region (France), and the DGIST R\&D Program of the Ministry of Science, ICT and Future Planning (17-BT-02) (South Korea).

\section{REFERENCES}

${ }^{7}$ C. J. Cochrane, J. Blacksberg, M. A. Anders, and P. M. Lenahan, Sci. Rep. 6, 37077 (2016).

${ }^{2}$ K. Kapser, M. Weinberger, W. Granig, and P. Slama, Giant Magnetoresistance (GMR) Sensors (Springer, 2013), pp. 133-156.

${ }^{3}$ R. S. Gaster, L. Xu, S.-J. Han, R. J. Wilson, D. A. Hall, S. J. Osterfeld, H. Yu, and S. X. Wang, Nat. Nanotechnol. 6, 314 (2011).

${ }^{4}$ G. Rizzi, M. Dufva, and M. F. Hansen, Lab Chip 17, 2256 (2017).

${ }^{5}$ F. Terki, A. Bousseksou, Q. H. Tran, S. Kamara, C. Kim, K. W. Kim, and Ph. Gandit, EP 2872911B1 (25 May 2016).

${ }^{6}$ F. Terki, Q. H. Tran, S. Kamara, G. Felix, A. Bousseksou, C. G. Kim, and K. W. Kim, WO2017140877A1 (2017).

${ }^{7}$ C. Goldberg and R. E. Davis, Phys. Rev. 94, 1121 (1954).

${ }^{8}$ T. Q. Hung, F. Terki, S. Kamara, M. Dehbaoui, S. Charar, B. Sinha, C. Kim, P. Gandit, I. A. Gural'Skiy, G. Molnar, L. Salmon, H. J. Shepherd, and A. Bousseksou, Angew. Chem. Int. Ed. 52, 1185 (2013).

${ }^{9}$ S. Kamara, T. Q. Hung, V. Davesne, G. Félix, L. Salmon, K. Kim, C. G. Kim, A. Bousseksou, and F. Terki, Adv. Mater. 29, 1703073 (2017).

${ }^{10}$ T. Q. Hung, S. Oh, B. Sinha, J.-R. Jeong, D.-Y. Kim, and C. Kim, J. Appl. Phys. 107, 09E715 (2010).

${ }^{11}$ X. J. Li, C. Feng, X. Chen, J. Y. Zhang, Y. W. Liu, S. L. Jiang, Y. Liu, M. H. Li, and G. H. Yu, J. Magn. Magn. Mater. 381, 386 (2015).

${ }^{12}$ M. D. Cubells-Beltrán, C. Reig, D. R. Muñoz, S. I. P. C. de Freitas, and P. J. P. de Freitas, IEEE Sensors J. 9, 1756 (2009).

${ }^{13} \mathrm{M}$. Vopalensky and A. Platil, IEEE Trans. Mag. 49, 136 (2013).

${ }^{14}$ G. Malinowski, M. Hehn, F. Montaigne, E. Jouguelet, and A. Schuhl, Appl. Phys. Lett. 84, 1204 (2004).

${ }^{15}$ H. Kagiwada, R. Kalaba, N. Rasakhoo, and K. Spingarn, Numerical Derivatives and Nonlinear Analysis (Springer Science \& Business Media, 2013).

${ }^{16}$ J. P. King, J. N. Chapman, M. F. Gillies, and J. C. S. Kools, J. Phys. D: Appl. Phys. 34, 528 (2001).

17T. McGuire and R. Potter, IEEE Trans. Mag. 11, 1018 (1975).

${ }^{18}$ X. Martí, B. G. Park, J. Wunderlich, H. Reichlová, Y. Kurosaki, M. Yamada, H. Yamamoto, A. Nishide, J. Hayakawa, H. Takahashi, and T. Jungwirth, Phys. Rev. Lett. 108, 017201 (2012).

${ }^{19}$ S. Lee, S.-K. Bac, S. choi, H. Lee, T. Yoo, S. Lee, X. Liu, M. Dobrowolska, and J. K. Furdyna, Sci. Rep. 7, 1115 (2017). 